\title{
Repeated text in unrelated passages: Repetition versus meaning selection effects
}

\author{
Celia M. Klin, April M. Drumm, and Angela S. Ralano \\ State University of New York, Binghamton, New York
}

\begin{abstract}
Despite previous findings, Klin, Ralano, and Weingartner (2007) found transfer benefits across unrelated passages. After processing an ambiguous phrase in Story A that was biased toward its sarcastic meaning, readers were more likely to interpret the identical phrase in Story B as sarcastic, even though it contained no disambiguating information. In the present experiments, we found both repetition effects (a benefit for the lexical items) and meaning selection effects (a benefit for the selected meaning of the phrase) with short delays between Stories A and B; with longer delays, only repetition effects were found. Whereas decreasing the elaboration of the phrase eliminated both effects, moving the disambiguating context from before to after the phrase eliminated meaning selection effects only. We conclude that meaning selection effects, which are based on conceptual overlap, are more sensitive to context changes and less robust than repetition effects, which are based on both perceptual and conceptual overlap.
\end{abstract}

A central goal of research in discourse comprehension is to determine what information is included in a reader's memory representation. One way to address this question is to investigate rereading, or text repetition, effects. Participants might be asked to read an entire passage twice, or individual words within a passage might be repeated from a first to a second reading. The logic of this methodology is that any information from the first reading that influences the second reading must have been stored in memory. Influence on the second reading has been demonstrated in a number of ways, including an increase in reading speed during the second reading (e.g., Levy et al., 1995; Raney \& Rayner, 1995), improved performance on a secondary task, such as word stem completion (e.g., MacLeod, 1989), and most recently, evidence that the interpretation of the repeated text has been influenced by the first reading (Klin, Ralano, \& Weingartner, 2007).

Interestingly, repetition effects are most often limited to conditions in which the context remains consistent across the two processing episodes. Consider a few examples. Levy et al. (1995) found facilitation in reading time in a related condition, when two passages came from the same novel and, thus, shared characters and a story line, but they found no rereading benefit in a word overlap condition in which the two passages shared the same number of words as in the related condition, were written in the same genre, but did not share characters or a theme. Similarly, Levy and Burns (1990) presented multiparagraph passages that were identical across two readings, had the individual paragraphs reordered, had the sentences reordered, or had the words reordered. Although there were rereading benefits for the identical condition and the paragraph- reordered condition, there were limited rereading benefits for the sentence-reordered condition and no rereading benefits for the word-reordered condition. Finally, using ERPs as the dependent measure, Besson and Kutas (1993) found repetition effects (indicated by a decrease in N400 amplitude during a second reading) when words were presented a second time in their original sentence frame, but not when they were presented in a new sentence.

Studies in which word lists have been used have also shown that repetition benefits tend to be limited to conditions in which the context remains consistent across the two processing episodes. For example, using a perceptual identification task, Levy and Kirsner (1989) found a benefit for words that were both studied and tested as a part of a word list but found no benefit for words that were originally seen in the context of a passage and subsequently identified in a word list. Similarly, Oliphant (1983) found a reprocessing benefit for repeated words in a lexical decision task but found no reprocessing benefit when the words were first processed as part of the instructions for the experiment.

Why might rereading transfer benefits be limited in this way? According to an episodic account, repetition priming is the result of the reactivation of the initial processing episode (e.g., Tenpenny, 1995; for similar accounts, see also Hayman \& Jacoby, 1989; Jacoby, Baker, \& Brooks, 1989). Consistent with this, Levy et al. (1995) argued that the memory representation of a word is contextually bound, making it necessary to recruit the original reading episode in order to find repetition effects. Given this, only when the context - that is, the reading episode - is repeated will there be rereading benefits.

C.M. Klin, cklin@binghamton.edu 
Although most rereading studies have provided evidence that is consistent with an episodic account, there are some exceptions. For example, Carr, Brown, and Charalambous (1989) found evidence of abstract, or contextindependent, repetition effects when participants were instructed to read paragraphs aloud quickly and to clearly enunciate the words. During rereading, a repetition effect was found even when the word order was scrambled, suggesting that the representation of the words was not bound to the text representation. Although this provides support for a context-independent account, this support seems to be limited to conditions in which readers focus on wordlevel processing, rather than on comprehension.

In an attempt to account for both the abstract and episodic findings, Raney (2003) proposed a contextdependent representation model in which surface information and textbase information (van Dijk \& Kintsch, 1983) are represented abstractly, in a context-independent manner, whereas the situation model is represented in a context-dependent manner. On the basis of this, the model predicts that repetition effects should be context independent if a situation model has not been constructed-for example, when readers ignore the meaning of a text and focus on the individual lexical items. In contrast, when readers are focused on comprehension, repetition effects should be found only when the situation model is consistent across the two processing cycles.

Despite these arguments, Klin et al. (2007) hypothesized that there should be at least some conditions in which repetition effects are found across unrelated passages, even when readers are focused on comprehension. Why might this be? According to many memory models (e.g., Gillund \& Shiffrin, 1984; Hintzman, 1986; Ratcliff, 1978), inputs to memory should cause related concepts from all of memory to resonate in parallel. If we assume that text inputs act like any other inputs to memory, a repeated segment of text should be able to reactivate related information from all of long-term memory, including information from an unrelated text (e.g., McKoon \& Ratcliff, 1995; Myers \& O'Brien, 1998). Myers and O'Brien argued that although the majority of the reactivated concepts during reading will be from the episodic memory trace- that is, from the same passage - this is simply because there are usually more related concepts within a narrative than from elsewhere in memory, rather than because the representation of the words is episodically bound.

To determine whether text repetition effects actually require a shared situation model or are simply more probable when this is the case, Klin et al. (2007) created passage pairs that had no thematic overlap but shared a salient phrase. (For a similar study, see Collins \& Levy, 2007, who had "less related" passage pairs that shared several sentences.) Consider the passage pair in Table 1. There were two versions of Story A - a repeated version, which contained a repetition line, and a not-repeated version, which did not. Story A was followed by Story B, which shared one line with the repeated version of Story A. Importantly, Story A and Story B did not share characters, a setting, or a story line. The question involved the process-
Table 1

Sample Item From Experiment 1

\section{STORY A}

\section{Introduction}

Shortly after Valerie graduated from college, she moved into an apartment with her friend Eric. Yesterday Eric, worrying that Valerie would forget about the phone bill, left her a note advising her to pay the bills as soon as they came.

\section{Critical Information}

Repeated condition (repetition line). Valerie always paid the bills on time, and was really irritated that Eric felt the need to leave constant reminders. She really wanted to tell him off. So after she ate breakfast, she wrote an angry note to Eric about his nagging and added: "Thanks so very much for all your advice. Sure is nice to be treated like a 10 year old."

Not-repeated condition. Valerie had almost forgotten that the bill was due today and was really glad that Eric had left her a reminder. She really wanted to thank him. So after she ate breakfast, she wrote a thank you note to Eric for the reminder and added: "I really do appreciate your reminders. It makes it easy to pay the bills on time."

\section{Conclusion}

Then Valerie went for a jog around her neighborhood. She was running in a big marathon next month and knew that she would need to train every day if she had any chance of making it to the finish line.

\section{STORY B}

\section{Introduction}

Brian was invited to his friend Laura's birthday party. When Brian arrived, he saw Laura right away and starting chatting with her. He told her that he had a crush on one of his coworkers. He wasn't sure if the attraction was mutual. Laura told Brian that he should take a chance and ask her out. The next day Brian wrote an e-mail to Laura to let her know that he left his jacket at her house and added:

\section{Repetition Line \\ "And thanks so very much for all your advice."}

\section{Sarcasm Disambiguation Line}

He was irritated that she always gave him advice.

\section{Spillover Line}

He could never tell her about anything

\section{Conclusion}

without her trying to tell him what to do.

ing of the repetition line in Story B. Note that the phrase in the repetition line in Story B was ambiguous; in the sample passage, it is unclear whether Brian is being sincere or sarcastic. In contrast, in the repeated version of Story A, the repetition line is unambiguously sarcastic. (In the not-repeated version of Story A, the repetition line is absent.) The logic of the design was as follows: When the participants in the repeated condition read the repetition line in Story B, if they reactivated from memory the identical phrase from Story A, this should bias a sarcastic interpretation of the phrase. This should, in turn, lead to shorter reading times on the sarcasm disambiguation line in the repeated than in the not-repeated condition.

This is exactly what Klin et al. (2007) found; readers were more likely to interpret the repetition line in Story B as sarcastic if they had encountered the line in Story A. In Experiment 1, readers were more likely to respond "true" to a sarcasm-consistent statement (e.g., Brian did not ap- 
preciate Laura's advice) presented after Story B if they had read the repeated version of Story A rather than the not-repeated version. In Experiment 2, readers in the repeated condition were slower to read a sincere-consistent disambiguation line (e.g., Laura was relieved that Brian appreciated her advice) that followed the repetition line in Story B. In Experiment 3, readers were faster to read a sarcasm-consistent disambiguation line (e.g., He was irritated that she always gave him advice) in the repeated condition than in the not-repeated condition. These effects were found despite the fact that the sarcastic interpretation of the phrase was its subordinate meaning; Klin et al. (2007) found that readers judged this phrase to be sincere $89 \%$ of the time when there was no disambiguating context.

The primary goal of the present set of experiments was to gain a better understanding of the repetition effects found by Klin et al. (2007). More specifically, we asked what stimulus properties might have accounted for their finding evidence of context-independent repetition effects when others had not. Carr and Brown (1990) have argued that it is too simplistic to expect processing to be either abstract or episodic. Instead, "one should be asking whether a particular computation is abstract or episodic with respect to a particular stimulus property" (p. 732). Similarly, Klin et al. (2007) concluded that the extent to which a word or phrase reactivated concepts from a different context should be dependent on the same complex set of factors that influence memory retrieval more generally: the amount of overlap between the two readings, the delay between the first and second readings, the amount of attention readers pay to the repeated text, the magnitude of the change in the two contexts, and so on. Thus, to gain a better understanding of the contribution of different stimulus properties to abstract, or context-independent, repetition effects, in the present set of experiments, we examined the influence of two factors: (1) the delay between the first and second readings of the repeated phrase and (2) the salience of the repetition phrase in Story A.

An additional goal of these experiments was to contrast repetition effects and meaning selection effects. In Klin et al. (2007), shorter reading times on the sarcasm disambiguation line required not only a repetition effect as it is traditionally defined (reactivation from memory of the individual words in the phrase), but also a meaning selection effect (reactivation of the sarcastic meaning of the ambiguous phrase). In the present experiments, we asked whether the two effects have different underlying mechanisms and whether they are differentially influenced by manipulations of delay and salience. We expected that meaning selection effects might be less robust and, perhaps, more episodic in nature than repetition effects. If this were the case, we should be able to identify conditions in which a repetition effect is found across two independent passages but a meaning selection effect is not.

Why might this be? Repetition effects are based on both perceptual and conceptual overlap, whereas meaning selection effects are based only on conceptual overlap. In the passages used by Klin et al. (2007), readers encountered a set of lexical items in the repeated phrase in Story B that was identical to those they had encountered in Story A. In addition to sharing meaning, there was a complete overlap of the physical features from the first encounter to the second. In contrast, readers' interpretation of the phrase in Story A is simply conceptual in nature; there is no perceptual representation of the sarcastic meaning. Given this, we expect that repetition benefits for the lexical items in the phrase should last longer than repetition effects for the selected meaning of the phrase.

There is evidence from across a number of domains that priming in tasks that involve a perceptual representational system (Tulving \& Schacter, 1990) can persist across retention intervals that are very long and that are considerably longer than those based solely on conceptual processes. For example, in a rereading study, Collins and Levy (2007) had participants read two 15- to 17 -sentence passages that shared 7 sentences. Repetition effects, defined as faster reading for the repeated sentences, were found across a 24-h delay when the passages were highly related. Kolers (1976) found shorter rereading times for typographically inverted text when the first reading had taken place 13-15 months earlier. Outside the domain of text, there is similar evidence of long-term priming effects, sometimes across exceptionally long delays. For example, Sloman, Hayman, Ohta, Law, and Tulving (1988) compared word fragment completion rates for words that had been previously studied with those for words that had not. They found greater completion rates for the studied words 16 months after the initial study episode ("through two Christmases and two New Years"; p. 237), even though each word was studied for only about $7 \mathrm{sec}$ during the initial presentation.

In contrast with the longevity of repetition priming, we expect that meaning selection effects will be less robust. Although there are no studies examining meaning selection effects for ambiguous phrases, there is a fairly extensive literature examining meaning selection effects for ambiguous words. The studies do not provide an entirely clear picture, but the findings certainly suggest that the duration of meaning selection effects is more modest than that of repetition effects. Although Gorfein, Berger, and Bubka (2000) found some priming for the selected meaning of a homograph 30 trials later, most other studies have reported durations that are considerably shorter. For example, Simpson and Kang (1994) presented homograph primes along with targets that selected one meaning of the homograph (e.g., bank-save). When the homograph was repeated on a second trial, there was no benefit when the selected meaning was the same across the two trials (e.g., bank-money), as compared with a control. This was true at both short and long intervals. (On the other hand, they did find inhibition for the nonselected meaning of the homograph.) Examining homographs embedded in sentences, Gernsbacher, Robertson, and Werner (2001) found a benefit if the selected meaning of a homograph was repeated, but the longest duration that they examined was seven intervening sentences. And finally, in a passage- 
reading task, Binder and Morris (1995) measured eye movements and found a cost associated with changing the meaning of a homograph from its first to second reading; however, there was only one sentence intervening between the two presentations. Thus, although the exact duration of meaning selection effects for ambiguous words is a matter of some debate (and is entirely unknown for ambiguous phrases), it seems plausible that the duration is shorter than that for repetition effects.

Finally, in addition to the prediction that repetition effects and meaning selection effects differ in their duration, meaning selection effects may be more sensitive to changes in context. In contrast with lexical items that have a permanent representation in memory, memory for a phrase may be more tied to the episode in which it was encountered. Given this, when there is a change in context between the first and the second readings, the selected meaning of the phrase may be less accessible. Consistent with this, Binder and Morris (1995) found that when a homograph in a passage changed meaning from its first to its second encounter, the slowdown on the second encounter continued into a posttarget region only when the passage topic remained consistent. When there was a topic change between the two encounters with the word, the slowdown for the changed meaning disappeared quickly.

\section{EXPERIMENT 1}

We began by replicating the findings from Experiment 3 in Klin et al. (2007). The first step was to demonstrate that repetition and meaning selection effects can be found across independent passages. This replication was necessary in part because, in Experiments 2, 3, 4, and 5, we tested for these effects under less optimal conditions and, thus, predicted some null effects.

\section{Method}

Participants. Eighty-two undergraduates at the State University of New York at Binghamton participated as part of the requirement for an introductory psychology course. The data were eliminated for 4 participants who did not follow instructions and for an additional 3 participants because their data were so variable. Therefore, the data analyses were based on 75 participants.

Materials. The present experiment used the materials from Klin et al. (2007; see Table 1 for a sample item). There were 14 experimental items, with each item consisting of a pair of independent stories, Story A and Story B. The items were presented in either a repeated or a not-repeated condition. Whereas Story B was identical across the two conditions, there were two versions of Story A: repeated and not repeated. Both versions of Story A began with an introduction that described an interaction between two characters. This was followed by a critical information section. In the repeated version of this section, one character, the message writer, felt negatively about the interaction. Because of this, the message writer left a sarcastic note for the other character, the addressee. The sarcastic note consisted of two sentences (e.g., "Thanks so very much for all your advice. Sure is nice to be treated like a 10 year old."). The first sentence included the phrase that was repeated in Story B (the repetition line). This sentence was written so that if it appeared in isolation, it would be ambiguous. That is, without context, the phrase "thanks so very much for all your advice" could be interpreted as either sarcastic or sincere. However, to ensure that the readers interpreted this phrase sarcastically, there was disambiguating context both before and after the repetition line. The sentence that preceded the repetition line explicitly stated that the message writer was angry or unhappy (e.g., she wrote an angry note to Eric) and the sentence following the repetition line was unambiguously sarcastic (e.g., "Sure is nice to be treated like a 10 year old.").

In the not-repeated version of Story A, no information was given in the critical information section regarding how the interaction between the characters was perceived by the message writer. Furthermore, the repetition line was not included. In its place, the message writer left a note that was unambiguous and sincere (e.g., "I've left a pot of soup cooking on the stove on low heat. Can you turn it off at 10:00?"). The critical information sections in the repeated and the not-repeated versions were matched for length. Finally, the conclusion provided a two-sentence story ending. The conclusion was identical in both versions and was neutral with regard to the repetition line.

Story B was identical in the repeated and not-repeated conditions. Furthermore, it was semantically and thematically unrelated to Story A; the two stories did not share a story line, characters, or a setting. However, like Story A, Story B consisted of a message writer and an addressee. The introduction described the interaction between the two characters. This was followed by the repetition line, which, again, was a note that the message writer left for the addressee. This was identical to the repetition line in the repeated version of Story A. However, in contrast to Story A, the phrase was presented in a neutral context and was, therefore, ambiguous; it was unclear whether the note was intended to be sarcastic or sincere. Next was a sarcasm disambiguation line that made it clear that the addressee had interpreted the repetition line as sarcastic. Finally, there was a spillover and conclusion line that served to wrap up the story.

Note that for each of the 14 experimental items, a different critical information section was used. That is, although a sarcastic note was always described in the repeated version of Story A, a different ambiguous phrase was used for each experimental passage (e.g., "Thanks so much for all your advice; The place sure does look trendy; That local talent is something else"). Twenty-six filler items were also presented. Fifteen of the fillers had a phrase in quotes to make them similar to the experimental items. However, in order to minimize the number of ambiguous phrases in the experiment, none of the phrases in quotation marks were ambiguous. Immediately after the last line of Story B and at the end of 19 of the 26 filler items, a comprehension question was presented to ensure that the participants were reading carefully. For half of the comprehension questions, the correct answer was yes.

Design. For each participant, experimental items were randomly assigned to the repeated and not-repeated conditions, with two constraints: (1) Each participant read half of the experimental items in each version, and (2) each version was presented to half of the participants. Filler items were interspersed among the experimental items, and the order of presentation was the same for all the participants.

Procedure. The participants were tested individually in sessions that lasted approximately $45 \mathrm{~min}$. They were instructed to read each story for comprehension. The items were presented on a computer monitor. The participants controlled the presentation of the text with a line advance key on a response box. Each keypress caused the current line of text to be erased and the next line to be presented. Between stories, the phrase "Press Advance For the Next Story" appeared at the center of the monitor.

Immediately after the last line of Story B and after the last line of 19 of the 26 filler items, a keypress caused the current line to be erased and the string "XXX" to appear in the center of the screen. The participants did not know that they were at the end of the story until this cue appeared. After $500 \mathrm{msec}$, this string was replaced by a comprehension question. The participants responded to the question by pressing either a "yes" or "no" key on the response box. Following the participants' response, the question was erased from the screen. The participants were given two breaks during the experiment. To familiarize the participants with the procedure, they were given four practice items before beginning the experiment. 


\section{Results and Discussion}

Statistical analyses were performed both with participants as a random effect variable $\left(t_{1}\right)$ and with items as a random effect variable $\left(t_{2}\right)$. An alpha level of .05 was used for all the analyses. Furthermore, for all the analyses, outliers were discarded (Tukey, 1977). For the present experiment, this eliminated $7.9 \%$ of the data. The mean reading times for the repetition line and sarcasm disambiguation line are presented in Table 2. Reading times for the repetition line in Story B were shorter in the repeated condition than in the not-repeated condition $\left[t_{1}(74)=4.1, S E M=\right.$ $\left.56.1 ; t_{2}(13)=3.3, S E M=54.4\right]$. Thus, reading times were shorter when the repetition line was read for the second time in the repeated condition than for the first time in the not-repeated condition. Furthermore, consistent with Klin et al.'s (2007) findings, reading times for the sarcasm disambiguation line were also shorter in the repeated condition than in the not-repeated condition $\left[t_{1}(74)=2.5\right.$, $\left.S E M=46.7 ; t_{2}(13)=2.2, S E M=64.9\right]$. There were no reading time differences for the spillover line $(p \mathrm{~s}>.6)$.

When the participants in the repeated condition read the repetition line in Story B, they reactivated both the words of the phrase from memory and its sarcastic meaning. This occurred even though Story A and Story B were episodically distinct and even though the readers had no reason to believe that Story A and Story B made up a single trial. Furthermore, Klin et al. (2007) demonstrated that the slow-

Table 2

Experiments 1-5: Reading Times (in Milliseconds) for Repetition and Sarcasm Disambiguation Lines (Story B)

\begin{tabular}{|c|c|c|}
\hline Condition & Repetition & $\begin{array}{c}\text { Sarcasm } \\
\text { Disambiguation }\end{array}$ \\
\hline \multicolumn{3}{|c|}{$\begin{array}{l}\text { Experiment 1: Replication of Klin, } \\
\text { Ralano, and Weingartner (2007) }\end{array}$} \\
\hline Not repeated & 1,945 & 2,384 \\
\hline Repeated & 1,716 & 2,270 \\
\hline Difference & $229^{*}$ & $114^{*}$ \\
\hline \multicolumn{3}{|c|}{$\begin{array}{l}\text { Experiment 2: One Intervening Passage } \\
\text { Between Story A and Story B }\end{array}$} \\
\hline Not repeated & 1,883 & 2,580 \\
\hline Repeated & 1,775 & 2,436 \\
\hline Difference & $108^{* *}$ & $144^{* *}$ \\
\hline \multicolumn{3}{|c|}{$\begin{array}{c}\text { Experiment 3: Two Intervening Passages } \\
\text { Between Story A and Story B }\end{array}$} \\
\hline Not repeated & 1,910 & 2,327 \\
\hline Repeated & 1,777 & 2,345 \\
\hline Difference & $133^{* *}$ & -18 \\
\hline \multicolumn{3}{|c|}{$\begin{array}{c}\text { Experiment 4: One Line of Elaboration } \\
\text { Removed From Story A }\end{array}$} \\
\hline Not repeated & 1,859 & 2,601 \\
\hline Repeated & 1,909 & 2,631 \\
\hline Difference & -50 & -30 \\
\hline \multicolumn{3}{|c|}{$\begin{array}{l}\text { Experiment 5: Disambiguating Information } \\
\text { Follows the Repetition Line in Story A }\end{array}$} \\
\hline Not repeated & 1,734 & 2,217 \\
\hline Repeated & 1,590 & 2,250 \\
\hline Difference & $144^{*}$ & -33 \\
\hline
\end{tabular}

down on the sarcasm disambiguation line in Story B was not due simply to the presence of any sarcastic information in Story A; in a control experiment, they found that readers did not interpret the Story B repetition line as sarcastic when an unrelated sarcastic phrase was present in Story A.

\section{EXPERIMENT 2}

In Experiments 2 and 3, we investigated the influence of delay on repetition and meaning selection effects. In Experiment 1, the repeated phrase in Story B was read relatively soon after the same line had been processed in Story A. Although passages were moderately long, only approximately 120 words separated the two presentations of the repetition line. And because the repetition line was so salient in Story A - being presented in quotation marks, being used in its figurative sarcastic meaning, and being surrounded by several lines of elaboration - it may have been relatively active in memory when the identical line was encountered in Story B.

To provide a test of the effect of delay, in Experiment 2, we inserted a passage between Story A and Story B. This not only increased the time between subsequent readings of the repetition line, but also added another context change between Story A and Story B. Whereas Story A and Story B had some similarities in their structure - both involved an interaction between two characters and both involved one of the characters' leaving a note- the intervening passage did not possess these characteristics.

Although repetition effects have been demonstrated across relatively long delays (e.g., Collins \& Levy, 2007; Kolers, 1976), meaning selection effects have not. Thus, there may be a repetition effect in Story B (shorter reading times for the repetition line in the repeated condition) but no meaning selection effect (no reading time difference for the sarcasm disambiguation line).

\section{Method}

Participants. Sixty-nine undergraduates at the State University of New York at Binghamton participated as part of the requirement for an introductory psychology course. The data were eliminated from 3 participants who did not follow instructions. Therefore, the data analyses were based on 66 participants.

Materials. The experimental materials were identical to those used in Experiment 1. The only change was that a 13-line filler passage was presented between Story A and Story B for each of the experimental pairs. Each intervening filler passage had a phrase in quotation marks to make it similar to the experimental items. However, in order to minimize the number of ambiguous phrases in the experiment, none of the phrases were ambiguous. In addition to the 14 filler passages that appeared between Story A and Story B, 7 filler passages were presented. These 7 filler passages varied in length, and 2 of them contained a phrase that was ambiguous and could be interpreted as either sarcastic or sincere. Four of these appeared as practice passages at the beginning of the experiment, and the other 3 were presented between experimental pairs (i.e., after Story B and before Story A). This was done to ensure that no pattern could be discerned by the participants. Comprehension questions were presented after each passage - Story A, Story B, and filler passages.

Design and Procedure. The design and procedure were identical to those used in Experiment 1, except for the placement and number of the filler passages. 


\section{Results and Discussion}

The mean reading times for the repetition line and the sarcasm disambiguation line are presented in Table 2 ( $8.3 \%$ of the data were eliminated as outliers). Despite the presence of an intervening passage between Story A and Story B, readers continued to reactivate from memory the lexical items in the repetition line when reading Story B. This led to shorter reading times for the repetition line in the repeated condition than in the not-repeated condition. The difference was reliable by participants $\left[t_{1}(65)=\right.$ $2.07, S E M=52.4]$ but not by items $\left[t_{2}(13)=1.5, S E M=\right.$ 64.2, $p=.15$ ]. Despite the nonsignificant items effect, ${ }^{1}$ the pattern of reading times for the sarcasm disambiguation line provide further evidence that the repeated line was reactivated from memory when it was presented in Story B. Reading times were also shorter for the sarcasm disambiguation line in the repeated condition than in the not-repeated condition; this difference was significant by participants $\left[t_{1}(65)=2.6, S E M=56.1\right]$ and significant by items in a one-tailed test $\left[t_{2}(13)=1.8, S E M=65.9, p=\right.$ $.10]$. Finally, the effect continued onto the spillover line, with reading times again being shorter in the repeated version (1,879 vs. $1,978 \mathrm{msec})$. This difference was reliable by participants $\left[t_{1}(65)=2.3, S E M=41.7\right]$, but not by items $\left[t_{2}(13)=1.2, S E M=72.2, p=.27\right]$.

Despite evidence demonstrating that the repetition of the original reading episode, or situation model, is needed for repetition benefits to occur, in Experiment 2, not only did we find repetition effects across independent passages, this occurred even when Story A and Story B were not contiguous. The intervening filler passage increased the amount of time between subsequent presentations of the repetition line and provided an additional change in context. Furthermore, meaning selection effects were found as well. Although the phrase was used in its subordinate meaning in Story A, and although there was no biasing context for the phrase in Story B, the subordinate meaning was again selected in Story B, despite the delay between the two presentations.

Might it be argued that readers did not reactivate the sarcastic interpretation of the phrase until they attempted to integrate the sarcasm disambiguation line into the passage? On the basis of the present data, this possibility cannot be ruled out. However, Klin et al. (2007) conducted an experiment in which the sarcasm disambiguation line was replaced with a sincere disambiguation line (e.g., Laura was relieved that Brian appreciated her advice). When readers encountered this line, there was no reason for them to reactivate the sarcastic interpretation at that time. Despite this, reading times were longer for the sincere disambiguation line in the repeated than in the not-repeated condition, indicating that the sarcastic interpretation of the repetition line had already been reactivated.

\section{EXPERIMENT 3}

In Experiment 3, we asked whether repetition and meaning selection effects would be found with two passages intervening between Story A and Story B. There is some evidence of text repetition effects across a 24-h delay (Collins \& Levy, 2007). However, this was done with passages that had substantial overlap; 7 sentences were repeated across passages that were 15-17 sentences in length, and thus, there were many opportunities for readers to retrieve the original processing episode. The question was whether a single overlapping phrase would be retrieved from memory when two unrelated passages intervened between Story A and Story B.

\section{Method}

Participants. Fifty-three undergraduates at the State University of New York at Binghamton participated as part of the requirement for an introductory psychology course. The data were eliminated from 3 participants who did not follow instructions. Therefore, the data analyses were based on 50 participants.

Materials. The experimental materials were identical to those used in Experiments 1 and 2. The only change from Experiment 2 was that now two filler passages, instead of one, were presented between Story A and Story B. The first intervening filler passage was the same 13-line filler passage used in Experiment 2, and the second was a new 10-line filler passage. Thus, each of the 14 experimental trials consisted of Story A, Filler 1 (13 lines), Filler 2 (10 lines), Story B. In order to make the filler passages similar to the experimental passages, 15 of the Filler 2 passages contained a phrase or a sentence in quotation marks (which was not sarcastic or ambiguous), and 2 additional filler passages contained a phrase that was ambiguous and could be interpreted as sarcastic or sincere. In addition to the 28 intervening filler passages that appeared between Story A and Story B, there were 3 filler passages presented as practice passages at the beginning of the experiment. No additional filler passages were presented between experimental pairs, because the experiment was rather long - 28 experimental passages (14 Story A-Story B pairs) and 31 filler passages. Yes/no comprehension questions were presented after each passage.

Design and Procedure. The design and procedure were identical to those used in Experiment 2, except for the number and placement of the filler passages.

\section{Results and Discussion}

The mean reading times for the repetition line and the sarcasm disambiguation line are presented in Table 2 (7.3\% of the data were eliminated as outliers). The results were quite clear: Reading times were shorter for the repetition line in the repeated condition than in the not-repeated condition. Although this effect was not reliable by items, the effect size was impressive: Reading times were $133 \mathrm{msec}$ shorter in the repeated condition $(95 \%$ confidence interval: $17-236 \mathrm{msec})\left[t_{1}(49)=2.3, S E M=54.4\right.$, and $t_{2}(13)=$ $1.2, S E M=95.7, p=.24]$. Despite the nonsignificant items effect, we suspect that there was a repetition effect. First, a similar-sized effect was found for the repetition line in Experiments 1 and 2. And second, as was noted earlier, the items analyses with this design were underpowered; thus, the nonsignificant items analysis was likely to have been a Type II error. In contrast with the findings for the repetition line, with two intervening passages between Story A and Story B, readers no longer read the sarcasm disambiguation line more quickly in the repeated condition than in the not-repeated condition $(p s>.7)$. There were also no spillover line differences $(p s>.7)$.

The contrasting results for the repetition line and the sarcasm disambiguation line are intriguing. If the readers 
reactivated their memory representation of the repetition line when processing this line in Story B, why were reading times not shorter for the sarcasm disambiguation line as well? The most plausible explanation is that when the readers encountered the repetition line in Story B, they reactivated the lexical items in the phrase from memory without reactivating its sarcastic interpretation. With two passages intervening between Story A and Story B, the sarcastic interpretation of the phrase may no longer have been easily retrievable from memory. Particularly given that the sarcastic interpretation of the repetition line was the subordinate interpretation, this interpretation may have been lost from memory relatively quickly. Thus, the readers seem to have reactivated either all or some of the lexical items from memory without reactivating the sarcastic meaning of the phrase.

So what can we conclude thus far? On the basis of the results of Experiment 2, it is clear that repetition effects are not as fragile as we might have suspected. Even though Story A and Story B passages did not share a theme, characters, or a setting, repetition effects were found when the repeated phrase was presented after a significant delay, defined both by the amount of time between readings and by the amount and type of intervening text. Whether a repetition effect was found with two passages intervening between Story A and Story B is somewhat less clear, given the nonsignificant items effect. However, regardless of whether the repetition effect persisted across one versus two intervening passages, the following is clear. (1) Our finding of repetition effects across unrelated linguistic contexts was not dependent on the repeated phrases' being presented in contiguous passages. Once the repetition line from Story A was encoded into memory, the identical phrase in Story B was an effective retrieval cue, even though the phrases were in different contexts. (2) Meaning selection effects seem to be more influenced by a change in context - temporal context, in this case - than are repetition effects. Thus, as was predicted, "priming in perceptual tasks is theorized to be mediated by a perceptual representation system and priming that is mediated by this system appears to be less affected by long retention intervals than is priming based on conceptual processes" (Mitchell, 2006, p. 926).

\section{EXPERIMENT 4}

In Experiment 4, we changed our focus from delay to salience. If we assume that text inputs act like any other input to memory (cf. Gillund \& Shiffrin, 1984; Hintzman, 1986; Ratcliff, 1978), the degree to which related concepts in memory resonate should depend, in part, on their level of activation and on the number of semantic and conceptual features that overlap with the current text input. Thus, the probability of retrieving the Story A repetition line from memory should increase if it has been elaborated extensively. Elaboration of the repetition line in Story A should increase its salience, leading readers to attend more strongly to it. This, in turn, may increase the probability that the phrase will be encoded into memory, as well as increase its level of activation. In addition, elaboration provides additional propositions, which should lead the repeti- tion line to be more richly encoded into memory, making it easier to retrieve. Decreasing the amount of elaboration should therefore decrease the probability of finding either a repetition effect or a meaning selection effect.

In Experiment 4, we removed one sentence of elaboration from the critical information section of Story A. More specifically, we eliminated the sarcasm elaboration sentence that followed the repetition line in the repeated condition. For example, in contrast with the sample passage from Experiments 1, 2, and 3, Story A no longer contained the line, "Sure is nice to be treated like a 10 year old." Although this may have decreased the perceived sarcasm of the repetition line, it was still unambiguously sarcastic, given the surrounding context. To ensure that this was the case, the note left was explicitly described as being sarcastically intended, using words such as bitterly, sarcastic, or irritated. The question was whether a repetition and a meaning selection effect would still be found (see Table 3 for a sample passage).

Table 3

Sample Item From Experiment 4

\section{STORY A}

\section{Introduction}

Shortly after Valerie graduated from college, she moved into an apartment with her friend Eric. Yesterday Eric, worrying that Valerie would forget about the phone bill, left her a note advising her to pay the bills as soon as they came in.

\section{Critical Information}

Repeated condition (repetition line). Valerie always paid the bills on time, and was really irritated that Eric felt the need to leave constant reminders. She really wanted to tell him off. So after she ate breakfast, she wrote an angry note to Eric about his nagging and added bitterly: "Thanks so very much for all your advice."

Not-repeated condition. Valerie had almost forgotten that the bill was due today and was really glad that Eric had left her a reminder. She really wanted to thank him. So after she ate breakfast, she wrote a thank you note to Eric for the reminder and added: "I really do appreciate your reminders."

\section{Conclusion}

Then Valerie went for a jog around her neighborhood. She was running in a big marathon next month and knew that she would need to train every day if she had any chance of making it to the finish line.

\section{STORY B}

\section{Introduction}

Brian was invited to his friend Laura's birthday party. When Brian arrived, he saw Laura right away and starting chatting with her. He told her that he had a crush on one of his coworkers. He wasn't sure if the attraction was mutual. Laura told Brian that he should take a chance and ask her out. The next day Brian wrote an e-mail to Laura to let her know that he left his jacket at her house and added:

\section{Repetition Line}

"And thanks so very much for all your advice."

\section{Sarcasm Disambiguation Line}

He was irritated that she always gave him advice.

\section{Spillover Line}

He could never tell her about anything

\section{Conclusion}

without her trying to tell him what to do. 


\section{Method}

Participants. Forty-six undergraduates at the State University of New York at Binghamton participated as part of the requirement for an introductory psychology course.

Materials. Two changes were made to the experimental materials from Experiment 1. (1) One sentence was removed from the critical information section of Story A. This was the sarcastic elaboration sentence that followed the repetition line-the second sentence in quotation marks. In the repeated version, this sentence always reiterated the sarcastic intent of the repetition line. In the not-repeated version, this sentence always reiterated the sincere intent of the repetition line. (2) A word was added to the sentence preceding the repetition line in Story A (e.g., bitterly) to make it clear that the repetition line was intended sarcastically.

Design and Procedure. The design and procedure were identical to those used in Experiment 1.

\section{Results and Discussion}

The mean reading times for the repetition line and the sarcasm disambiguation line are presented in Table 2 ( $6.8 \%$ of the data were eliminated as outliers). In contrast with the previous results, there was no difference in reading times for the repetition line ( $p s>3)$. And, not surprisingly, with no repetition effect, there was also no meaning selection effect. Reading times did not differ for the sarcasm disambiguation line $(p \mathrm{~s}>.7)$. Finally, there were no posttarget differences.

By all indications, when readers processed the repetition line in Story B, it did not lead to its reactivation from memory. Simply removing one line of elaboration from Story A was sufficient to eliminate the effect. In the repeated version, not only was the repetition line in Story B not read more quickly than in the not-repeated condition, where it appeared for the first time, it was not interpreted as sarcastic. Whereas increasing the delay between the two presentations of the repetition line did not significantly influence the pattern of findings, a reduction in elaboration powerfully reduced both the repetition and the meaning selection effects.

Eliminating a line of elaboration from Story A may have reduced the likelihood that the repetition line was encoded into memory. In addition, even if the repetition line was encoded, the representation may have been more sparse, with fewer propositions and fewer interconnections, making retrieval less probable. Another possible effect of eliminating a line of elaboration was to reduce the perceived sarcasm of the repetition line. Although the repetition line was still unambiguously sarcastic, the sarcasm may have been less apparent with the elaboration line removed. And if the sarcastic meaning increased the salience of the phrase, this may have served to reduce the attention that the phrase received and the probability of its being encoded.

\section{EXPERIMENT 5}

In Experiment 4, we found that reducing the amount of elaboration surrounding the repetition line eliminated the repetition and meaning selection effects. In Experiment 5 , we reduced the sarcastic emphasis of the phrase without reducing the amount of elaboration. To do this, we changed the placement of the negative information in Story A. Consider the sample passage in Table 4. In contrast with the previous experiments, the negative information followed the repetition line rather than preceded it. Thus, at the point at which readers encountered the repetition line in Story A, it was ambiguous; it could have been interpreted as sarcastic or sincere. Furthermore, given the Klin et al. (2007) findings, we know that the default interpretation of the critical sentence is sincere; without any biasing context, the participants in Klin et al. (2007) judged the repetition line to be sincere $89 \%$ of the time. Thus, in the present experiment, we expected that the participants would initially interpret the repetition line in Story A as sincere in the majority of cases. However, the sentence that followed the repetition line made it clear that the intention of the message writer was sarcastic. Given this, there was no doubt that the readers would quickly come

Table 4

Sample Item From Experiment 5

\section{STORY A}

\section{Introduction}

Shortly after Valerie graduated from college, she moved into an apartment with her friend Eric. Yesterday Eric, worrying that Valerie would forget about the phone bill, left her a note advising her to pay the bills as soon as they came in.

\section{Critical Information}

Repeated condition (repetition line). After Valerie ate breakfast, she wrote a note to Eric and added: "Thanks so very much for all your advice. Sure is nice to be treated like a 10 year old." Valerie always paid the bills on time, and was really irritated that Eric felt the need to leave constant reminders. So she had really wanted to tell him off.

Not-repeated condition. After Valerie ate breakfast, she wrote a note to Eric and added: "I really do appreciate your reminders. It makes it easy to pay the bills on time." Valerie had almost forgotten that the bill was due today and was really glad that Eric had left her a reminder. So she had really wanted to thank him.

\section{Conclusion}

Then Valerie went for a jog around her neighborhood. She was running in a big marathon next month and knew that she would need to train every day if she had any chance of making it to the finish line.

\section{STORY B}

\section{Introduction}

Brian was invited to his friend Laura's birthday party. When Brian arrived, he saw Laura right away and starting chatting with her. He told her that he had a crush on one of his coworkers. He wasn't sure if the attraction was mutual. Laura told Brian that he should take a chance and ask her out. The next day Brian wrote an e-mail to Laura to let her know that he left his jacket at her house and added:

\section{Repetition Line}

"And thanks so very much for all your advice."

\section{Sarcasm Disambiguation Line}

He was irritated that she always gave him advice.

\section{Spillover Line}

He could never tell her about anything

\section{Conclusion}

without her trying to tell him what to do. 
to understand that the note was intended sarcastically. However, if the phrase was not initially encoded sarcastically - without, for example, the prosody associated with sarcasm - the sarcastic meaning might not be as strongly associated with the phrase and, thus, would perhaps not be retrieved during rereading.

A number of results were possible. First, the results might replicate those found in Experiments 1 and 2. The readers might continue to encode the repetition line in Story A as sarcastic, given the disambiguating information in the line that followed it. Furthermore, because the amount of elaboration had not been reduced, in the repeated condition reading times might be shorter for both the repetition line and the sarcasm disambiguation line in Story B. A second possibility was that the results would mimic those found in Experiment 4: If the sarcastic meaning was not initially encoded, this might make the repetition line in Story A less memorable, reducing the likelihood that it would be encoded and retrieved, eliminating both the repetition effect and the meaning selection effect. And finally, the results might mimic those in Experiment 3: If the sarcastic meaning was less strongly associated with the repetition line in Story A, when the readers processed the repetition line in the repeated version of Story B, it might reactivate from memory the lexical items in the phrase without reactivating its sarcastic meaning. This would lead to shorter reading times on the repetition line, but not on the sarcasm disambiguation line.

\section{Method \\ Participants. Forty-three undergraduates at the State University of New York at Binghamton participated as part of the requirement for an introductory psychology course. The data were eliminated from 2 participants who did not follow instructions. Therefore, the data analyses were based on 41 participants. \\ Materials. The experimental materials were based on those used in Experiment 1, but with the sentences in the critical information section reordered. The critical information section began with the sentence that contained the repetition line. In addition, in the re- peated version, any words that indicated that the note was intended sarcastically (e.g., angrily, bitterly) were removed so that the phrase was temporarily ambiguous. The repetition line was followed by the remainder of the critical information section, which made it clear that the note was intended sarcastically. Importantly, the critical information section in Story A was almost identical to the critical information section in the previous experiments; except for some minor wording changes, it was simply the order of the sentences that was changed. Therefore, in contrast with the manipulation in Experi- ment 4 , the amount of elaboration was not reduced.}

Design and Procedure. The design and procedure were identical to those used in Experiment 1.

\section{Results and Discussion}

The mean reading times for the Story B repetition line and the sarcasm disambiguation line are presented in Table 2 (5.9\% of the data were eliminated as outliers). The pattern of results was quite clear: Reading times were significantly shorter for the repetition line in the repeated condition than in the not-repeated condition $\left[t_{1}(41)=2.53\right.$, $\left.S E M=57.1 ; t_{2}(13)=2.10, S E M=78.67, p=.056\right]$. However, there was no meaning selection effect: Reading times were no shorter for the sarcasm disambiguation line in the repeated than in the not-repeated condition $\left[t_{1}(41)=\right.$ $0.54, S E M=61.55, p=.59 ; t_{2}(13)=0.115, S E M=86.83$, $p=.91]$. Posttarget reading times also showed no evidence of a meaning selection effect ( $p s>.2)$.

It appears that rearranging the order of the information in the critical information section influenced processing in an interesting way, eliminating the meaning selection effect without affecting repetition effects. Although the readers were faster to read the repetition line when they read it a second time, indicating that they had retrieved the words in the phrase, they were no more likely to interpret it as sarcastic in the repeated condition than in the not-repeated condition. Why might this have been? It has been found that when biased ambiguous words are read in a neutral context, the dominant meaning becomes available first (e.g., Duffy, Kambe, \& Rayner, 2001). Because the disambiguating context was presented after the repetition line in Story A, the repetition line was ambiguous. Thus, the sincere interpretation should have been available first. And although readers quickly learned that the repetition line was intended sarcastically, on the basis of the disambiguating context that immediately followed, there was no reason for the readers to go back and fully reprocess the phrase-for example, adding a sarcastic intonation to the words. Because of this, the repetition line may not have been as likely to be encoded as sarcastic, or the sarcastic meaning may have been less strongly associated with the phrase.

\section{GENERAL DISCUSSION}

Despite the extensive evidence demonstrating that rereading benefits are limited to conditions in which the context remains consistent across the two processing episodes (e.g., Levy \& Burns, 1990; Levy et al., 1995), Klin et al. (2007) found text repetition effects across independent narratives. In the present experiments, we asked what factors contributed to this finding of context-independent repetition effects, when other studies have shown contextdependent repetition effects and when several accounts of rereading (Carr \& Brown, 1990; Raney, 2003) predict that when readers' attention is focused on passage comprehension or building a situation model, there should be no repetition effects across independent passages.

We started by replicating the findings of Klin et al. (2007), demonstrating that at least when conditions are optimal for memory retrieval, a change in the situation model does not prevent repetition effects. Although there may be an episodic component to the memory representation, with retrieval of concepts from within an episode being more probable than the retrieval of concepts from a different episode, this is simply one factor influencing the probability of successful retrieval. In Experiment 1, Story A and Story B had no situation model overlap, but the probability of finding repetition effects was optimized in other ways: The repeated text was a phrase, rather than an individual word; in Story A, the repetition line had been extensively elaborated; it was part of a dialogue; the phrase was used in its subordinate, sarcastic meaning; it was repeated across two contiguous passages; and so on. In the present set of 
experiments, we asked whether context-independent repetition effects are limited to these ideal conditions. Given a memory-based model of text processing, the extent to which a word or phrase reactivates concepts from the same narrative should depend on factors that are the same as those that influence the success of memory retrieval more generally. To begin to answer this question, we examined the influence of delay and salience.

In Experiments 2 and 3, we asked whether repetition effects and meaning selection effects would be found if the repeated text was encountered in noncontiguous passages. These experiments allowed us to determine whether retrieval was possible over a longer time span and with interference from an intervening passage. In addition, we examined the prediction that repetition effects would persist across longer delays than would meaning selection effects. This prediction stems largely from the fact that repetition effects are based on both perceptual and conceptual overlap, whereas meaning selection effects are based only on conceptual overlap. An additional goal of these experiments was to ensure that the context-independent repetition effects found in Experiment 1 were not dependent on readers' treating Story A and Story B as episodically related - for example, as consisting of a single trial. Although this was unlikely, adding one or two unrelated filler passages between Story A and Story B made this possibility implausible.

Impressively, in Experiment 2, when the readers processed an ambiguous phrase that had been previously encountered two passages earlier, not only did they retrieve the phrase from memory but also they retrieved their sarcastic interpretation of it. The presence of the repetition line two passages earlier was sufficient to influence readers' interpretation of the repetition line in Story B, causing them to be more likely to interpret it as sarcastic in the repeated condition than in the not-repeated condition, despite the fact that the default interpretation of the phrase was sincere.

In Experiment 3, when two passages intervened between Story A and Story B, there was a repetition effect (although not reliable in the items analysis) but no effect on the sarcasm disambiguation line. The readers seem to have retrieved from memory at least some of the words from the repetition line, but not the communicative intent of the phrase - that is, the sarcasm. (Although it is possible that the readers retrieved the sarcastic interpretation but failed to use it in interpreting the repetition line in Story B, this explanation seems unlikely.)

Although our results do not allow us to determine exactly what was retrieved from memory in Experiment 3, the combined results of Experiments 2 and 3 do allow us to conclude that our finding of context-independent repetition effects was not dependent on the readers' inferring that contiguous passages made up an experimental pair. In addition to ruling out this strategic explanation, the finding of rereading effects across a delay indicates that transfer benefits can be found with a substantial change in context. When the readers processed the repetition line in Story B, because of its overlap with information stored in memory, it served as an effective retrieval cue, automatically causing the words in the phrase to become reactivated. This occurred even though the first presentation of the phrase had been embedded in an unrelated passage. This provides evidence that the surface features in Story B - that is, the actual words in the repetition line-were neither glued to the situation model (Raney, 2003) nor contextually bound (Levy et al., 1995).

Why did the intervening passages interfere with the retrieval of the sarcastic interpretation but not the retrieval of the surface information - that is, the words themselves? One possibility is that during the first reading of the repetition line, there was priming of an abstract representation of the words in the phrase, such as their logogens (Morton, 1969). As a result, the input thresholds for these words were lowered, making them faster to access during the second presentation. This might have been true for either all of the words in the phrase or for simply a subset of the words. A related possibility is that during the rereading of the repetition line, the entire phrase, rather than some individual words, was retrieved from memory; however, only the sincere meaning of the phrase was accessed. This assumes that when Story A was read, the readers settled on the sarcastic meaning of the phrase only after encoding the literal meaning. Consistent with this idea, there is some evidence that figurative meanings are accessed more slowly than their literal counterparts (e.g., Gibbs, 1990; Onishi \& Murphy, 1993).

Why were the meaning selection effects lost when there was a delay? The mechanisms underlying long-term meaning selection effects remain a matter of some debate and have been addressed only for ambiguous words, not phrases. One proposal is that long-term priming of the selected meaning is the result of the inhibition of the alternative meanings (e.g., Gernsbacher, 1990; Simpson \& Kang, 1994; Swinney, 1981). An alternative proposal (Gorfein, 2001; Gorfein \& Brown, 2007) is that meaning selection involves reweighting the attributes of an ambiguous word in a way that is consistent with the selected meaning. It is this reweighting that leads to long-term priming for the selected meaning.

Either of these possibilities is plausible for ambiguous phrases as well: In Story A, the readers could have suppressed the sincere interpretation of the phrase, or they might have weighted the attributes of the phrase in a way that was consistent with its sarcastic interpretation. For example, the prosody associated with sarcasm may have been represented and weighted heavily. Given either idea - inhibition or reweighting of attributes - it is not surprising that after a delay, the selected, sarcastic meaning became less available. Although it is not clear whether meaning selection involves transient suppression or longer lasting inhibition (Gorfein \& Brown, 2007), at some point the activations of the inhibited meanings should return to their baseline levels, as should the weights of the attributes of the phrase. What the present work demonstrates is that the phrase meaning returns to baseline more quickly than the individual lexical items that make up the phrase. This is not surprising, given that there are examples of very 
long-term priming effects (e.g., Sloman et al., 1988) and of text repetition effects across a 24-h delay (Collins \& Levy, 2007), whereas there has been no evidence of this type of long-term meaning selection effect.

It is interesting to consider the results of Experiment 3 in light of research examining the influence of context on memory. In a study in which the remember-know paradigm was used to examine memory for faces (Gruppuso, Lindsay, \& Masson, 2007), a context change lowered participants' recollection while leaving their feelings of familiarity intact (e.g., Mandler, 1980). This finding provides another framework for understanding our results: When two passages intervened between Story A and Story B, the repetition line may have remained familiar to the readers, accounting for the faster reading speeds on the repetition line in the repeated version. In contrast, the intervening passages may have interfered with the readers' recollection of the details of the original episode. And without being able to recollect Story A, the readers were unable to access the sarcastic interpretation of the phrase, leading to a lack of a meaning selection effect on the disambiguation line.

In Experiments 4 and 5, we turned our attention from the influence of delay to the influence of the salience of the repetition line. In Experiment 4, we removed one line of elaboration from the critical information section of Story A. In addition to simply reducing the amount of information associated with the repetition line, this reduced the number of sarcastic propositions in the passage. Despite this, the repetition line remained unambiguously sarcastic in Story A. Although the manipulation was relatively subtle, the effects were not: Whereas adding an entire passage between Story A and Story B did not influence repetition or meaning selection effects, removing a single line of elaboration eliminated both of them entirely. Consistent with this, it has been found that reducing elaboration by even a single word can significantly influence processing, decreasing the speed of memory retrieval (Albrecht \& Myers, 1998) or decreasing the probability of retrieval (Klin, Weingartner, Guzmán, \& Levine, 2004).

What role did elaboration play? The simplest possibility is that reducing the elaboration influenced the encoding of the repetition line. There were fewer propositions related to the information in the repetition line, and there were fewer propositions reinforcing the sarcastic interpretation. This may have decreased the probability that the repetition line was encoded by decreasing its salience, as well as the amount of attention it received. Furthermore, this may have decreased the strength of the perceived sarcasm. A reduction in elaboration may have led to a memory representation that was less richly interconnected, making the repetition line in Story B a less effective retrieval cue to memory (e.g., Myers \& O’Brien, 1998). Another possibility is that when the repetition line was elaborated, this increased salience caused the readers to focus their attention on the lexical items making up the phrase. According to Carr and Brown's (1990) level-of-focal-attention hypothesis, when readers focus on comprehension, there should be a large contribution from text-level, or context-dependent, processing. However, if readers focus their attention on processing individual lexical items, there will be a large contribution from lexical, or context-independent, processing. If the elaboration increased the readers' attention to the individual lexical items making up the phrase, this would explain why a decrease in elaboration eliminated the context-independent repetition effects.

In Experiment 5, salience was manipulated by changing the order of the elaboration. In contrast with all of the other experiments, the sarcastic meaning of the repetition line was not available until after the phrase had been processed. Thus, it should have initially been encoded as sincere, given that the default interpretation of the phrase was sincere (Klin et al., 2007). And, although the disambiguating context followed immediately after the repetition line, the readers may not have gone back and fully reprocessed the repetition line. For example, the prosody associated with sarcasm may not have been encoded into the memory representation, given that the readers did not initially "hear" the phrase as sarcastic. This should have altered the way in which the phrase was encoded into memory.

Although we know relatively little about the form that text representations take, recent evidence suggests that the representation may include some characteristics of spoken language. Alexander and Nygaard (2008) had participants listen to an audio recording of one of two talkers, who were the supposed authors of a narrative that the participants subsequently read. For the participants who had been told that the narrative was written by the faster of the two talkers, reading times were shorter, both when they read the narrative aloud and when they read it silently. Although this manipulation included an explicit audio portion and speed, rather than other aspects of prosody such as intonation, was examined, the intriguing possibility is raised that various aspects of prosody are included in the linguistic representation during reading, despite the fact that no phonological information is actually present in the signal. Thus, the distinctive prosody associated with the sarcastic utterance may have been included in the readers' representations when the disambiguating context preceded the ambiguous phrase. But regardless of the way in which sarcasm is encoded - propositionally versus in some sort of phonological representation - we assume that the features that marked the dialogue as sarcastic were reduced or entirely missing from the readers' representations in Experiment 5, when the disambiguating context followed the phrase. However, because it was only the sarcastic interpretation that was affected by the reordering of the disambiguating information, the readers' ability to retrieve the words of the phrase from memory was not affected.

In conclusion, we have demonstrated that text repetition effects can be abstract, rather than episodic, and that these context-independent effects can be found even with a substantial delay between the first and second encounters. In contrast, meaning selection effects appear to be less robust. Manipulations of the elaboration of the repeated text indicate that when two passages do not share a theme, characters, or setting information, the repeated text must be salient for repetition effects to occur. Although the 
present experiments examined only the influence of elaboration, we suspect that other factors affecting the salience of the repeated text were also critical - the length of the phrases; their figurative, sarcastic meaning; the fact that they appeared in dialogue; and so on. To continue working toward an understanding of the nature of readers' memory representation, an understanding is needed of the influence of a wide range of stimulus and reader characteristics on transfer benefits.

\section{AUTHOR NOTE}

Portions of these data were reported at the 16th Annual Meeting of the Society for Text and Discourse, Minneapolis, MN. We thank Deborah Berk, Gina Choi, Meredith Jones, Heather Karmin, Alicia Kosiba, Steven Kovacs, Robyn Mierzejewski, and John Sentigar for their assistance with data collection. And thanks to Kristin Weingartner for comments on an earlier draft of the manuscript. Correspondence concerning this article should be addressed to C. M. Klin, Department of Psychology, State University of New York, Binghamton, NY 13902-6000 (e-mail: cklin@binghamton.edu).

\section{REFERENCES}

Albrecht, J. E., \& Myers, J. L. (1998). Accessing distant text information during reading: Effects of contextual cues. Discourse Processes, 26, 87-107.

Alexander, J. D., \& NygaArd, L. C. (2008). Reading voices and hearing text: Talker-specific auditory imagery in reading. Journal of Experimental Psychology: Human Perception \& Performance, 34, 446-459. doi:10.1037/0096-1523.34.2.446

Besson, M., \& Kutas, M. (1993). The many facets of repetition: A cued-recall and event related potential analysis of repeating words in same versus different sentence contexts. Journal of Experimental Psychology: Learning, Memory, \& Cognition, 19, 1115-1133. doi:10.1037/0278-7393.19.5.1115

Binder, K. S., \& Morris, R. K. (1995). Eye movements and lexical ambiguity resolution: Effects of prior encounter and discourse topic. Journal of Experimental Psychology: Learning, Memory, \& Cognition, 21, 1186-1196. doi:10.1037/0278-7393.19.5.1115

CARr, T. H., \& Brown, J. S. (1990). Perceptual abstraction and interactivity in repeated oral reading: Where do things stand? Journal of Experimental Psychology: Learning, Memory, \& Cognition, 16, 731738. doi:10.1037/0278-7393.16.4.731

Carr, T. H., Brown, J. S., \& Charalambous, A. (1989). Repetition and reading: Perceptual encoding mechanisms are very abstract but not very interactive. Journal of Experimental Psychology: Learning, Memory, \& Cognition, 15, 763-778. doi:10.1037/0278-7393.15.5.763

Collins, W. M., \& Levy, B. A. (2007). Text repetition and text integration. Memory \& Cognition, 35, 1557-1566.

Duffy, S. A., Kambe, G., \& RAYner, K. (2001). The effect of prior disambiguating context on the comprehension of ambiguous words: Evidence from eye movements. In D. S. Gorfein (Ed.), On the consequences of meaning selection: Perspectives on resolving lexical ambiguity (pp. 27-43). Washington, DC: American Psychological Association. doi:10.1037/10459-002

GeRnSBACHER, M. A. (1990). Language comprehension as structure building. Hillsdale, NJ: Erlbaum.

Gernsbacher, M. A., Robertson, R. R. W., \& Werner, N. K. (2001). The costs and benefits of meaning. In D. S. Gorfein (Ed.), On the consequences of meaning selection: Perspectives on resolving lexical ambiguity (pp. 119-137). Washington, DC: American Psychological Association. doi:10.1037/10459-007

Gibis, R. W. (1990). Comprehending figurative referential descriptions. Journal of Experimental Psychology: Learning, Memory, \& Cognition, 16, 56-66. doi:10.1037/0278-7393.16.1.56

Gillund, G., \& ShifFrin, R. M. (1984). A retrieval model for both recognition and recall. Psychological Review, 91, 1-67. doi:10.1037/0033 $-295 X .91 .1 .1$

GorfEIN, D. S. (2001). An activation-selection view of homograph dis- ambiguation: A matter of emphasis? In D. S. Gorfein (Ed.), On the consequences of meaning selection: Perspectives on resolving lexical ambiguity (pp. 157-173). Washington, DC: American Psychological Association. doi:10.1037/10459-009

Gorfein, D. S., Berger, S., \& BubKA, A. (2000). The selection of homograph meaning: Word associations when context changes. Memory \& Cognition, 28, 766-773.

Gorfein, D. S., \& Brown, V. R. (2007). Saying no to inhibition: The encoding and use of words. In D. S. Gorfein \& C. M. MacLeod (Eds.), Inhibition in cognition (pp. 103-124). Washington, DC: American Psychological Association. doi:10.1037/11587-006

Gruppuso, V., Lindsay, D. S., \& Masson, M. E. J. (2007). I'd know that face anywhere! Psychonomic Bulletin \& Review, 14, 1085-1089.

Hayman, C. A. G., \& Jacoby, L. L. (1989). Specific word transfer as a measure of processing in the word-superiority paradigm. Memory \& Cognition, 17, 125-133.

Hintzman, D. L. (1986). "Schema abstraction" in a multiple-trace memory model. Psychological Review, 93, 411-428. doi:10.1037/0033 -295X.93.4.411

JACOBY, L. L., BAKER, J. G., \& BROOKS, L. (1989). Episodic effects on picture identification: Implications for theories of concept learning and theories of memory. Journal of Experimental Psychology: Learning, Memory, \& Cognition, 15, 275-281. doi:10.1037/0278-7393.15.2.275

Klin, C. M., Ralano, A. S., \& Weingartner, K. M. (2007). Repeating phrases across unrelated narratives: Evidence of text repetition effects. Memory \& Cognition, 35, 1588-1599.

Klin, C. M., Weingartner, K. M., Guzmán, A. E., \& Levine, W. H. (2004). Readers' sensitivity to linguistic cues in narratives: How salience influences anaphor resolution. Memory \& Cognition, 32, 511522 .

KoLERs, P. A. (1976). Reading a year later. Journal of Experimental Psychology: Human Learning \& Memory, 2, 554-565. doi:10.1037/0278 $-7393.2 .5 .554$

LeVy, B. A., \& Burns, K. I. (1990). Reprocessing text: Contributions from conceptually driven processes. Canadian Journal of Psychology, 44, 465-482. doi:10.1037/h0084265

Levy, B. A., Campsall, J., Browne, J., Cooper, D., Waterhouse, C., \& Wilson, C. (1995). Reading fluency: Episodic integration across texts. Journal of Experimental Psychology: Learning, Memory, \& Cognition, 21, 1169-1185. doi:10.1037/0278-7393.21.5.1169

LEVY, B. A., \& KIRSNER, K. (1989). Reprocessing text: Indirect measures of word and message level processes. Journal of Experimental Psychology: Learning, Memory, \& Cognition, 15, 407-417. doi:10.1037/0278-7393.15.3.407

MacLeOD, C. M. (1989). Word context during initial exposure influences degree of priming in word fragment completion task. Journal of Experimental Psychology: Learning, Memory, \& Cognition, 15, 398-406. doi:10.1037/0278-7393.15.3.398

Mandler, G. (1980). Recognizing: The judgment of previous occurrence. Psychological Review, 87, 252-271. doi:10.1037/0033 $-295 X .87 .3 .252$

McKoon, G., \& Ratcliff, R. (1995). The minimalist hypothesis: Directions for research. In C. A. Weaver, S. Mannes, \& C. R. Fletcher (Eds.), Discourse comprehension: Essays in honor of Walter Kintsch (pp. 97-116). Hillsdale, NJ: Erlbaum.

Mitchell, D. B. (2006). Nonconscious priming after 17 years: Invulnerable implicit memory? Psychological Science, 17, 925-929. doi:10.1111/j.1467-9280.2006.01805.x

Morton, J. (1969). Interaction of information in word recognition. Psychological Review, 76, 165-178. doi:10.1037/h0027366

Myers, J. L., \& O'Brien, E. J. (1998). Accessing the discourse representation during reading. Discourse Processes, 26, 131-157.

Oliphant, G. W. (1983). Repetition and recency effects in word recognition. Australian Journal of Psychology, 35, 393-403. doi:10.1080/ 00049538308258751

ONISHI, K. H., \& MurPhy, G. L. (1993). Metaphoric reference: When metaphors are not understood as easily as literal expressions. Memory \& Cognition, 21, 763-772.

RANEY, G. E. (2003). A context-dependent representation model for explaining text repetition effects. Psychonomic Bulletin \& Review, 10, $15-28$

RANEY, G. E., \& RAYNER, K. (1995). Word frequency effects and eye 
movements during two readings of a text. Canadian Journal of Experimental Psychology, 49, 151-172.

RATCLIFF, R. (1978). A theory of memory retrieval. Psychological Review, 85, 59-108. doi:10.1037/1196-1961.49.2.151

Simpson, G. B., \& KANG, H. (1994). Inhibitory processes in the recognition of homograph meanings. In D. Dagenbach \& T. H. Carr (Eds.), Inhibitory processes in attention, memory, and language (pp. 359381). New York: Academic Press.

Sloman, S. A., Hayman, C. A. G., Ohta, N., Law, J., \& Tulving, E. (1988). Forgetting in primed fragment completion. Journal of Experimental Psychology: Learning, Memory, \& Cognition, 14, 223-239. doi:10.1037/0278-7393.14.2.223

SWINNEY, D. (1981). Lexical processing during sentence comprehension: Effects of higher order constraints and implications for representation. In T. Myers, J. Laver, \& J. M. Anderson (Eds.), The cognitive representation of speech (pp. 201-210). Amsterdam: North-Holland.

Tenpenny, P. L. (1995). Abstractionist versus episodic theories of repetition priming and word identification. Psychonomic Bulletin \& Review, 2, 339-363.

TuKeY, J. W. (1977). Exploratory data analysis. Reading, MA: AddisonWesley.

Tulving, E., \& Schacter, D. L. (1990). Priming and human memory systems. Science, 247, 301-306. doi:10.1126/science.2296719
VAN DiJK, T. A., \& KInTSCH, W. (1983). Strategies of discourse comprehension. New York: Academic Press.

\section{NOTE}

1. Given the design of the experiments in this article, in conjunction with the limitations on the participants' attention span, it was not possible to have a sufficient number of items for high power for the items analyses. Even with only 7 items in each of the two conditions (repeated and not repeated), this involved 14 experimental items. Furthermore, because each item consisted of two passages (Story A and Story B), this meant an average of 300 words per item. An additional 26 filler passages were needed -3 as practice in the beginning of the experiment and 23 more interspersed throughout the 28 experimental passages to prevent the participants from catching on to the manipulation. Because this added up to a hefty 54 passages (with even more for Experiment 3 ), a more desirable 10 or 12 passages per condition was not feasible. Given this, some nonsignificant items analyses were inevitable.

(Manuscript received June 16, 2008; revision accepted for publication February 16, 2009.) 